\title{
Value-at-risk under ambiguity aversion
}

Rossella Agliardi

Correspondence:

rossella.agliardi@unibo.it Department of Mathematics, University of Bologna, Viale Filopanti, 5, Bologna, Italy

\begin{abstract}
This study explored the effects of ambiguity on the calculation of Value-at-Risk (VaR) using a mathematical model based on the theory of Choquet-Brownian processes. It was found that while a moderate degree of ambiguity aversion yields a higher value for VaR and Expected Shortfall (ES), the result can be reversed in a deeply ambiguous environment. Additionally, some sufficient conditions are provided for the preservation of this effect under various forms of risk aggregation. This study offers a new perspective to full awareness on capital requirement calculation as requested by international regulation.
\end{abstract}

Keywords: Choquet-Brownian motion, Risk measures, Ambiguity aversion

\section{Background}

One of the key issues in prudential regulation is the appropriate calculation of the so-called capital requirement. Since 1993, when the Bank of International Settlements (BIS) announced its intention to introduce a capital requirement for market risk, and especially after 1996, when regulators began to allow and promote the use of internal models to measure risk exposures, quantile measures such as Value-at-Risk (VaR) have become very common. The VaR framework, originally introduced by RiskMetrics for market risk, has been extended to valuate the credit risk embedded in loans and privately traded bonds as in CreditMetrics (see Gupton et al. 1997). The Basel Committee Internal Models Approach prescribes that a bank must have a model in place to capture specific risks in its regulatory capital, and capital charges are specified in terms of VaR-based calculations.

The VaR measure is defined as the minimum loss that will occur with a certain probability. For example, a confidence level of $99 \%$ requires capital levels to be set so that the estimated probability of insolvency is lower than $1 \%$ per year. In the European Solvency II measures, the economic capital an insurance company must hold to guarantee a one-year ruin probability of at most $0.5 \%$ is named the Solvency Capital Requirement (SCR) and is calculated using VaR techniques. Moreover, some aggregation formulas have been proposed to combine several forms of risk (see EIOPA-14-322 2014). Since using VaR to set capital requirements does not provide information about the potential size of losses exceeding the VaR measure, measures such as the Conditional Tail Expectation (CTE), Tail-Value-at-Risk (TVaR), and Expected Shortfall (ES) ${ }^{1}$ have recently been proposed as well. ES at the $99 \%$ confidence level is the average loss given that the loss falls within the worst $1 \%$ part of the distribution. The advantage of this measure over VaR is discussed in Artzner et al. (1999), and it has been adopted in some regulatory directives (e.g., the Swiss Solvency Test).

(c) The Author(s). 2018 Open Access This article is distributed under the terms of the Creative Commons Attribution 4.0 International License (http://creativecommons.org/licenses/by/4.0/), which permits unrestricted use, distribution, and reproduction in any medium, provided you give appropriate credit to the original author(s) and the source, provide a link to the Creative Commons license, and indicate if changes were made. 
While regulators and scholars have made great efforts to differentiate and properly quantify different risk exposures, the incorporation of a more elusive form of risk (i.e. ambiguity and its effect on the calculation of capital requirements) remains an unexplored issue. The measurement of a specific risk presupposes that the probabilities are known or can be estimated with confidence. Meanwhile, ambiguity refers to incalculable uncertainty and pertains to realistic situations where there is subjective uncertainty about the probability measure governing the outcomes. A precise definition of ambiguity is offered in Epstein and Schneider (2010).

Agents may differ in their prior beliefs and attitudes toward ambiguity. This may lead to distortions and deviations from 'true' estimates and eventually result in different decisions even though the same risk-management techniques are adopted. The inconvenience of excessive reliance on a single probabilistic model was highlighted in the Turner Review (2009), as it became especially salient after the global financial crisis. The crisis of 2007-2009 brought attention to how subjective perceptions of uncertainty and behavior affect the real economies and markets. In turbulent economic times, ambiguity aversion provides better explanations than risk aversion (e.g., Guidolin and Liu 2016). Meanwhile, various studies have linked ambiguity and financial crises (f.e. Boyarchenko 2010) or have shown that incorrect beliefs arising from probability weighting or misestimation have a sizable effect on investors' utility gains Hens and Rieger (2014). To avoid the disincentivizing effects of an obscure environment some non-model-based methods have been proposed to measure risk capital (see Ghamani 2015, for an application to CCP risk capital, for example).

In this study, we investigated the effect of ambiguity aversion on the most common risk measures by focusing on the case of Gaussian distributions. We chose Gaussian distribution because this assumption is behind many industry models for risk calculation and is implicit in many standard formulas and methods prescribed by international regulation. The incorporation of ambiguity was achieved by building on the method developed by Kast and Lapied (2010a, 2010b), which is theoretically founded and, at the same time, parsimonious enough to be easily calibrated to real-world data (see So 2017). However, as Agliardi et al. (2016) showed, the model relevant parameter can be mapped to a measure of average ambiguity aversion as proposed in Rieger et al. (2015), which provides a convincing proxy for ambiguity aversion bias.

Roughly speaking, under the adopted mathematical model the presence of ambiguity deforms the probability distribution throughout a parameter measuring the degree of ambiguity aversion or ambiguity seeking. Since deformation affects both the mean and the variance, the results are not easy to obtain and demand a careful analysis. We show that a moderate degree of ambiguity aversion always demands higher capital requirements, but in certain extreme situations, the presence of this form of uncertainty may decrease the calculated capital requirement.

The mathematical setting is presented in Section "5. Conclusion". Section 3 discusses the effect of ambiguity aversion on the most common risk measures, while Section 4 investigates the issue of risk aggregation. 


\section{Modeling normal distributions under ambiguity}

Throughout this study, the relative changes in portfolio value were assumed to follow Brownian motion, that is, a normal distribution was adopted to apply a parametric method for the relevant risk measures. To capture the ambiguous environment, we adopted a Choquet-Brownian model as in Kast and Lapied (2010a, 2010b). The uncertain setting was modeled through capacity theory, which replaces the classical probabilistic framework. By capacity on a set of uncertain states, $\mathrm{S}$, we mean a set function $v$ such that $v(\mathrm{~S})=1, v(\varnothing)=0$ and $\mathrm{A} \quad \mathrm{B} \Rightarrow v(\mathrm{~A}) \leq$ $v(B)$. A capacity $v$ is convex if.

$$
v(F U G) \geq v(F)+v(G)-v(F \cap G) \forall F, G .
$$

It is concave if the reverse inequality holds. Convexity and concavity are interpreted in terms of ambiguity aversion or ambiguity seeking, respectively, by the decision maker. We refer to Schmeidler (1989) for the link between the convexity of capacities and a representation of ambiguity aversion.

In this context, expectations are defined as Choquet integrals: $E(X)=\int_{S} X d v$.

As a first step, Choquet random walks are defined. A binomial lattice is considered and for each state.

$s_{t}$ at time $t, 0 \leq t \leq T$, the possible successors at time $\mathrm{t}+1$ are denoted as $s_{t+1}{ }^{u}$ ('up' movement) and $s_{t+1}{ }^{d}$ ('down' movement). The capacity of "up" and a "down" movements is defined as follows:

$$
v\left(s_{t}{ }^{u} \mid s_{t}\right)=v\left(s_{t}{ }^{d} \mid s_{t}\right)=c,
$$

where $c, 0<c<1, c$ is a constant that represents the decision-maker's ambiguity regarding the likelihood of the states to come. Note that we adopted symmetric Choquet random walks: the case of asymmetric Choquet binomial trees was investigated in Agliardi (2017). The relevant parameter $c$ plays a crucial role in the model and can be extracted from market data, as shown, for example, in So (2017) where the formulas of Driouchi et al. (2015) were used. The absence of an ambiguity bias is represented by the anchor case $c=1 / 2$, because, in this status, we are reduced to the classical symmetric random walks, while $c<1 / 2(c>1 / 2)$ describes the situation of an ambiguity-averse (seeking) decision maker.

Kast and Lapied (2010b) showed that the symmetric discrete process defined above converges to a distorted generalized Brownian motion. More precisely, we quote the following result:

\section{Proposition 5.2 in Kast and Lapied (2010b)}

When the time interval converges toward 0 , the symmetric random walk defined above converges toward a general Wiener process with mean $m=2 c-1$ and variance $s^{2}=4 c(1-c)$.

To explore the effect of ambiguity on risk measures in portfolio values, we need to compare biased estimates with those obtained under an undistorted stochastic process of the form. 


$$
d X_{t}=\mu d t+\sigma d W_{t}
$$

where $W t$ is a standard Wiener process with respect to an original probability measure toward which the decision-maker is perfectly confident. Therefore, we start with an arithmetic Brownian motion to model the relevant economic variable, where the source of uncertainty is governed by a Wiener process. Now, we introduce model risk in the form of ambiguity, that is, we suppose that the decision maker is not perfectly confident about the extent to which the ABM properly represents the wealth dynamics. As discussed above, we start with symmetric Choquet random walks with conditional capacity c. To obtain a continuous time framework, we consider the limit process as the time interval between two consecutive movements tends to 0 . As specified in the abovementioned Proposition 5.2 (Kast and Lapied 2010b), the limit process is a distorted Brownian motion whose parameters depend on the ambiguity level, c. Thus, ambiguity is introduced in the continuous time model (1) and takes the form of a distortion of the original ABM. In the biased process, the original drift is replaced with $\mu+m \sigma$ and the volatility with $s \sigma$. Note that for fully ambiguity-averse decision-makers one has $-1<m<0$ and $0<s<1$, so $\mu+m \sigma<\mu$ and $0<s \sigma<\sigma$, because the ambiguity parameter, $c$, is restricted to $0<\mathrm{c}<1 / 2$ in the case of ambiguity aversion, which implies that $m<0$ and $s<1$. Here, drift and volatility are both reduced in comparison to the case of the absence of ambiguity biases. Thus the direction of the resulting effect of ambiguity aversion is not easy to guess, as it depends on the relative values of the relevant parameters.

In Fig. 1 below, the distortion of a standard normal distribution function is obtained by varying the base parameter $\mathrm{c}$ while keeping $c \leq 1 / 2$. Figure 1 is obtained by taking $\mu=0, \sigma=1$ in the unbiased distribution (blue curve) and then modifying drift and volatility according to various ambiguity levels. Empirical evidence confirms that ambiguity aversion is prevalent among decision-makers. For example, Trautman and Van De Kuilen (2013) report that there is a clear evidence that on

\section{Gaussian distribution under ambiguity}

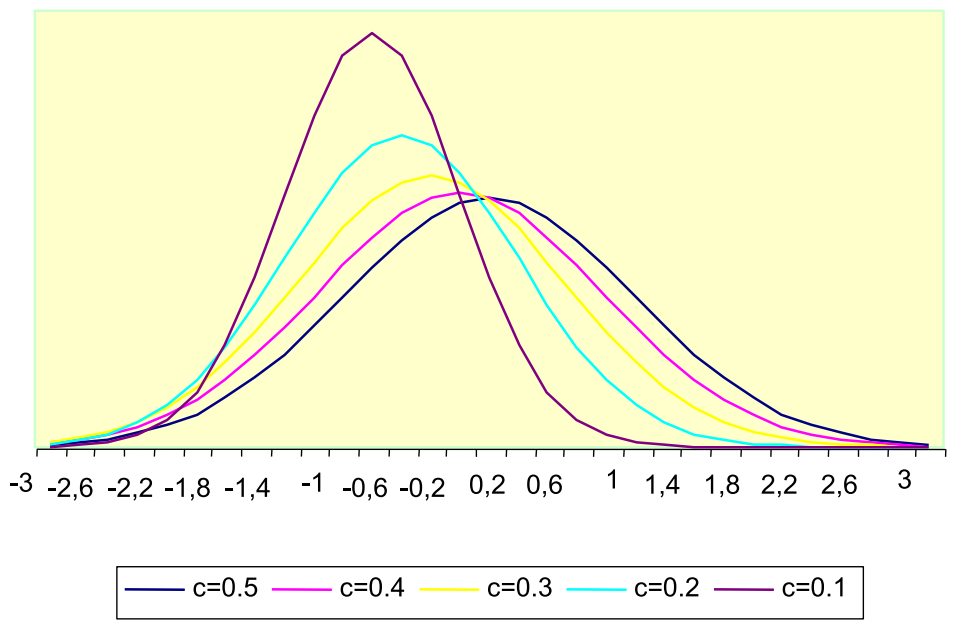

Fig. 1 Gaussian distribution under several ambiguity levels, c 
average ambiguity aversion is the typical qualitative finding'. Therefore, the subsequent analysis mainly focuses on the case $c \leq 1 / 2$.

\section{Value-at-risk and expected shortfall under ambiguity aversion}

Assume a portfolio has a risk exposure affording a risk management division to use Gaussian distributions. To fix ideas, in the base case we assume the profit and loss distribution has a zero mean value and a standard deviation of $10 \%$ during the prescribed time horizon. As the proof in Proposition 1 shows, our results are independent of the value of $\mu$, that is, they are robust to changes in the drift of the undistorted price process. In Table 1 Value-at-Risk calculation is performed on an initial portfolio value of 1000 units of currency by introducing ambiguity in the form of a parameter c, as explained in Section "5. Conclusion". The confidence levels are fixed at $99,99.5$ and $99.9 \%$ as these are relevant values in prudential regulation. For example, the Solvency Capital Requirement (SCR) is calibrated to the one-year VaR at the $99.5 \%$ confidence level, meaning that within the next year, the insurer's capital will only be insufficient to absorb unexpected losses in $0.5 \%$ of cases, or 1 out of 200 cases.

As a general rule, the capital requirement increases in the presence of a moderate degree of ambiguity, that is, when parameter c moves away from the base case, $c=0.5$; thus, ambiguity acts as an additional form of unfavorable uncertainty. Interestingly, when the ambiguity level becomes very high, the result is reversed and the calculated prudential capital may become even lower than in the unambiguous case. A possible explanation lies in the nature of ambiguity, which, on the one hand, shifts the loss distribution toward the unfavorable side but, on the other hand, hampers the role of "probabilistic" risk. In terms of risk measurement, the component related to expected risk is inflated in the presence of ambiguity, while the portion attributed to unexpected risk is slightly reduced: when the latter effect prevails in some extreme situations, the estimated amount of losses may even result in lower values.

In recent decades, risk professionals have started employing some coherent alternatives to VaR. Among them, Expected Shortfall is a natural choice whenever the concern is with the expected loss sustained in the portion of the unfortunate possibilities cut out by a fixed confidence level. Changing from VaR to ES to determine economic capital requirement is among the recommendations of the Basel Committee of Banking Supervision (BCBS 2013).

In Table 2, we present computations similar to those in Table 1 using the ES at various confidence levels.

The effect of ambiguity is similar for this risk measure - that is, an inverted U-shaped ES curve emerges as a function of the degree of ambiguity aversion parametrized by $c$.

Table 1 Value-at-Risk under several ambiguity levels

\begin{tabular}{llllll}
\hline $\mathrm{C}=$ & 0.5 & 0.4 & 0.3 & 0.2 & 0.1 \\
\hline VaR99\% & 232.63 & 247.93 & 253.21 & 246.11 & 219.58 \\
VaR99.5\% & 257.58 & 272.38 & 276.08 & 266.07 & 234.55 \\
VaR99.9\% & 309.03 & 322.78 & 323.23 & 307.22 & 265.42 \\
\hline
\end{tabular}


Table 2 Expected Shortfall under several ambiguity levels

\begin{tabular}{llllll}
\hline $\mathrm{C}=$ & 0.5 & 0.4 & 0.3 & 0.2 & 0.1 \\
\hline ES99\% & 266.52 & 281.14 & 284.27 & 273.22 & 239.91 \\
ES99.5\% & 289.19 & 303.35 & 305.05 & 291.35 & 253.52 \\
ES99.9\% & 336.69 & 349.89 & 348.58 & 329.35 & 282.01 \\
\hline
\end{tabular}

\section{Risk aggregation}

A crucial question in all risk assessment methodologies concerns how to aggregate the overall risk of a portfolio by combining the calculated risks of its individual components. For example, in the Solvency II paradigm, the market risk module comprises six submodules dealing with interest rate, equity, property, spread, currency risk, and the risk arising from market concentrations. The capital requirement for each submodule is determined based on prespecified scenarios, calibrated such that the resulting SCR should correspond to the VaR at the $99.5 \%$ confidence level over a one-year period. Finally, the overall capital requirement for market risk is obtained by combining individual SCRs through a predefined correlation matrix (see EIOPA-14-322 2014, pp. 138-139).

This section investigates the effect of ambiguity on the overall capital requirement of a portfolio by restricting the analysis to VaR. To simplify the analysis, we assume that all components are affected by the same degree of ambiguity, meaning that the modeller is the same for the several submodules.

\subsection{Analysis under a variance-covariance approach}

Consider a portfolio with a value of 1000 that consists of two assets whose return distributions are normal with a mean value of zero and standard deviations of $20 \%$ and $10 \%$. In Tables 3, 4 and 5, the calculation of the VaR on the total portfolio is performed for several values of the ambiguity level, $\mathrm{c}$, and by assuming a correlation $\rho=0$ in Table 3, $\rho=0.5$ in Table 4 , and $\rho=-0.5$ in Table 5 .

The increase in the VaR when a moderate level of ambiguity is introduced is confirmed, followed by a decline when $c$ becomes very low. Note that in the case of negative correlation, a stronger ambiguity aversion $(c=0.02)$ is needed to get a lower VaR level than in the unbiased value $(c=0.5)$.

Proposition 1 shows that the inversion in the direction of $\mathrm{VaR}$ is a general phenomenon. For simplicity's sake, we present the proof for the case of independent assets, although the result holds true in a more general situation, as specified in Remark 1.

Let $V_{\alpha}(c)$ denote the VaR of the whole portfolio at confidence level $\alpha$ and with a fixed level of ambiguity $c$.

In what follows, $\mathrm{N}($.$) denotes the cumulative distribution function for a standard$ Gaussian distribution.

Table 3 Value-at-Risk for a portfolio of two assets (correlation $\rho=0$ )

\begin{tabular}{lllllll}
\hline $\mathrm{C}=$ & 0.5 & 0.4 & 0.3 & 0.2 & 0.1 & 0.05 \\
\hline VaR99\% & 260.09 & 284.84 & 298.38 & 298.07 & 276.06 & 248.37 \\
VaR99.5\% & 287.99 & 312.17 & 323.94 & 320.39 & 292.79 & 260.53 \\
VaR99.9\% & 345.50 & 368.52 & 376.66 & 366.40 & 327.30 & 285.60 \\
\hline
\end{tabular}


Table 4 Value-at-Risk for a portfolio of two assets (correlation $\rho=0.5$ )

\begin{tabular}{lllllll}
\hline $\mathrm{c}=$ & 0.5 & 0.4 & 0.3 & 0.2 & 0.1 & 0.05 \\
\hline VaR99\% & 307.75 & 331.53 & 342.05 & 336.20 & 304.65 & 269.14 \\
VaR99.5\% & 340.75 & 363.87 & 372.30 & 362.60 & 324.45 & 283.53 \\
VaR99.9\% & 408.80 & 430.54 & 434.67 & 417.04 & 365.28 & 313.19 \\
\hline
\end{tabular}

\section{Proposition 1}

Let $\left(\theta_{1}, \ldots, \theta_{n}\right), \theta_{i} \geq 0$, be a portfolio of $n$ assets, whose returns $X_{i}, i=1, \ldots, n$, follow Choquet-Brownian motions with ambiguity parameter $c, 0<c<0.5$, and are independent. Then for every $\alpha>0.5$ there exists a critical value $c^{*}$ of the ambiguity parameter such that $V_{\alpha}(c) \leq V_{\alpha}\left(c^{*}\right)$ for any $c_{-}(0,0.5)$. Moreover, for any $\alpha>\mathrm{N}(\sqrt{n})$, there exists $\bar{c}$ $(0,0.5)$ such that $V_{\alpha}(c)<V_{\alpha}(0.5)$ for $\mathrm{c}<\bar{c}$.

\section{Proof}

Let $\sigma_{i}$ denote the standard deviation of $X_{i}, i=1, \ldots, n$, and let $\Xi=\left(\sum_{i=1}^{n} \theta_{i}^{2} \sigma_{i}^{2}\right)^{1 / 2}$. Then, $V_{\alpha}(c)$ is maximized when $(1-2 c) \sum_{i=1}^{n} \theta_{i} \sigma_{i}+2 \sqrt{c(1-c)} Q_{\alpha} \Xi$ is maximized, being $Q_{\alpha}$ the $\alpha$-quantile for the standard normal distribution. That is, $\mathrm{c}^{*}$ is obtained as the solution to.

$$
Q_{a} \Xi / \sum_{i=1}^{n} \theta_{i} \sigma_{i}=\frac{\sqrt{c^{*}\left(1-c^{*}\right)}}{0.5-c^{*}}
$$

As the function on the right-hand side ranges from 0 to $\mathbb{K}$, a solution $c^{*}$ is always found. $V_{\alpha}(c)<V_{\alpha}(0.5)$ is equivalent to $(1-2 c) \sum_{i=1}^{n} \theta_{i} \sigma_{i}+2 \sqrt{c(1-c)} Q_{\alpha} \Xi<Q_{\alpha} \Xi$. Let $\phi(c)$ $=\frac{1+2 \sqrt{c(1-c)}}{1-2 c} \geq 1$ for $0 \leq \mathrm{c}<0.5$. The above inequality can be written as $Q_{\alpha}>\phi(c) \sum_{i=1}^{n} \theta_{i} \sigma_{i} / \Xi$. As $\sum_{i=1}^{n} \theta_{i} \sigma_{i} / \Xi \leq \sqrt{n}$ by taking $\phi(\bar{c}) \leq Q_{\alpha} / \sqrt{n}$ one has $V_{\alpha}(c)<V_{\alpha}(0.5)$ for any $c, 0 \leq c \leq \bar{c}$.

\section{Remark 1}

This result is preserved in the general case of dependent assets. Let $\Theta^{t}=\left(\theta_{1}, \ldots, \theta_{n}\right), \mathbf{R}$ $=\left(\rho_{i j}\right)$ the correlation matrix and let $\Xi$ be replaced with $\Xi_{R}=$ $\left(\sum_{i=1}^{n} \theta_{i}^{2} \sigma_{i}^{2}+\sum_{i \neq j} \rho_{i j} \theta_{i} \theta_{j} \sigma_{i} \sigma_{j}\right)^{1 / 2}$ throughout the proof of Proposition 1 . Note that

Table 5 Value-at-Risk for a portfolio of two assets (correlation $\rho=-0.5$ )

\begin{tabular}{lllllll}
\hline $\mathrm{c}=$ & 0.5 & 0.4 & 0.3 & 0.2 & 0.1 & 0.02 \\
\hline VaR99\% & 201.47 & 227.40 & 244.65 & 251.17 & 240.88 & 200.41 \\
VaR99.5\% & 223.07 & 248.57 & 264.45 & 268.46 & 253.84 & 206.46 \\
VaR99.9\% & 267.62 & 292.22 & 305.28 & 304.10 & 280.57 & 218.93 \\
\hline
\end{tabular}




$$
V_{\alpha}(c)-V_{\alpha}(0.5)=(2 \sqrt{c(1-c)}-1) \Xi_{R} Q_{a}-(2 c-1) \sum_{i=1}^{n} \theta_{i} \sigma_{i}
$$

Thus $V_{\alpha}(c)$ is maximized when $c=c^{*}$, where $c^{*}$ is the solution to $Q_{\alpha} \Xi_{R} / \sum_{i=1}^{n} \theta_{i} \sigma_{i}$ $=\frac{\sqrt{c^{*}\left(1-c^{*}\right)}}{0.5-c^{*}}$. Moreover, $V_{\alpha}(c)<V_{\alpha}(0.5)$ is equivalent to $Q_{\alpha} \Xi_{R}>\phi(c) \sum_{i=1}^{n} \theta_{i} \sigma_{i}$, where $\phi(c)$ is as in the proof of Proposition 1.

If $\sum_{i, j} \rho_{i j} \geq 0$, by taking $\phi(\bar{c}) \leq Q_{\alpha} \sqrt{\sum_{i, j} \rho_{i j}} / n$ one has $V_{\alpha}(c)<V_{\alpha}(0.5)$ for any $c, 0 \leq c \leq \bar{c}$.

For example, if the assets exhibit a common correlation, ${ }^{2} \rho$, then the result holds for $\rho \geq 1 /(1-n)$. Indeed, $\Xi_{R}=\left(\sum_{i=1}^{n} \theta_{i}^{2} \sigma_{i}^{2}+\rho \sum_{i \neq j} \theta_{i} \theta_{j} \sigma_{i} \sigma_{j}\right)^{1 / 2}$ and $\Xi_{R}^{2} \geq \frac{(n-1) \rho+1}{n}\left(\sum_{i=1}^{n} \theta_{i} \sigma_{i}\right)^{2}$. Taking $\phi(\bar{c}) \leq Q_{\alpha} \sqrt{\frac{(n-1) \rho+1}{n}}$ one has $V_{\alpha}(c)<V_{\alpha}(0.5)$ for any c, $0 \leq c \leq \bar{c}$.

\section{Remark 2}

A similar argument can be applied to Expected Shortfall, because, in the case of normal distributions, it is multiplicative in the standard deviation and linear in the mean. More precisely, the ES for a profit and loss distribution with standard deviation $\sigma$ and mean $\mu$, at confidence level $\alpha$, is of the form: $\sigma E S_{\alpha}[N(0,1)]-\mu$. Therefore, the proof of Proposition 1 can be adapted to this risk measure with only a small modification.

\subsection{Extension to copula methods}

Thus far, the aggregation formula has been based on the assumption that the dependence between the distributions can be fully captured by linear correlations. In the mathematical literature, a number of examples can be found where the dependence between the marginal distributions is more complicated (see Embrechts et al. 2002). Overlooking the "true" dependence structure could lead to incorrect aggregated results, i.e. producing either an underestimation or an overestimation of the capital requirements at the aggregated level. To address this issue, (occurring, for example, in the case where there are tail dependencies), we can combine the marginal distributions throughout non-Gaussian copulas. As a first step, we must write the aggregate distribution (i.e. the distribution of the weighted sum of several random variables, each following a Choquet-Brownian motion). Then we compute a lower percentile of the aggregate distribution to estimate the VaR.

Copula theory provides an easier method for handling (otherwise) complex multivariate structures. The main idea behind this approach is that the marginals and the dependence function - the copula - can be modeled separately. The dependence relationship is determined by the copula, while scaling and shape depend on the marginals.

In mathematical terms, an n-dimensional copula is an n-dimensional distribution function restricted to $[0,1]^{n}$ with uniform- $(0 ; 1)$ marginals. For a given copula $\Phi$ and marginals $\mathrm{F} 1, \ldots$ Fn one has that 


$$
F\left(x_{1}, \ldots, x_{n}\right)=\Phi\left(F_{1}\left(x_{1}\right), \ldots, F_{n}\left(x_{n}\right)\right)
$$

is a distribution function with these marginals. Conversely, for a given joint distribution function $\mathrm{F}$ with marginals $\mathrm{F} 1, \ldots$ Fn there is always a copula $\Phi$ satisfying this relationship. Given a set of continuous marginal distributions, a unique copula function can be backed out:

$$
\Phi\left(u_{1}, \ldots, u_{n}\right)=F\left(F_{1}^{-1}\left(u_{1}\right), \ldots, F_{n}^{-1}\left(u_{n}\right)\right) .
$$

These results are known as Sklar's theorem (1959), and they provide motivation for modeling dependence structures throughout copula functions.

If $f_{i}\left(x_{i}\right), i=1, \ldots, \mathrm{n}$, denotes the marginal density function and $f\left(x_{1}, \ldots, x_{n}\right)$ is the joint density function, then differentiation yields.

$$
f\left(x_{1}, \ldots, x_{n}\right)=\phi\left(F_{1}\left(x_{1}\right), \ldots, F_{n}\left(x_{n}\right)\right) \prod_{i=1}^{n} f_{i}\left(x_{i}\right)
$$

where

$$
\phi\left(F_{1}\left(x_{1}\right), \ldots, F_{n}\left(x_{n}\right)\right)=\frac{\partial^{n} \Phi\left(F_{1}\left(x_{1}\right), \ldots F_{n}\left(x_{n}\right)\right)}{\partial F_{1} \ldots \partial F_{n}}
$$

When regarded as a function of $\left(u_{1}, \ldots, u_{n}\right)$ with $u_{i}=F_{i}\left(x_{i}\right), \phi$ is referred to as the copula density. For example, the bivariate normal copula density is of the form.

$$
\phi\left(u_{1}, u_{2}\right)=\frac{1}{\sqrt{1-\rho}} \exp \left[-\frac{\rho^{2} \xi_{1}^{2}+\rho^{2} \xi_{2}^{2}-2 \rho \xi_{1} \xi_{2}}{2-2 \rho^{2}}\right]
$$

where $\xi_{i}=N^{-1}\left(u_{i}\right), i=1,2$. This is the copula that is implied by a bivariate Gaussian copula. A more flexible copula is the Student's t copula, which is implied by multivariate $\mathrm{t}$-Student distribution and requires an additional parameter, $\mathbf{V}$, called the degrees of freedom. In contrast to the Gaussian copula, the Student's t copula exhibits both upper and lower tail dependence. Another example, belonging to the Archimedean family, is the Clayton copula density function, which in the bivariate case takes the form of

$$
\phi\left(u_{1}, u_{2}\right)=(a+1)\left(u_{1}^{-a}+u_{2}^{-a}-1\right)^{-2-1 / a} u_{1}^{-a-1} u_{2}^{-a-1}
$$

When $a>0$, the Clayton copula exhibits lower tail dependence. We referred to Nelsen (1999) for a thorough treatment of copula theory.

In what follows, we adopt Gaussian distributions for the marginals. This is because we need to compare the results with those above, and an explicit formula for the distorted process under ambiguity is available in this case. We denote by $X_{i}$ the random variable modeling the profits and losses on the ith asset in a portfolio and assume that losses are represented in the left tail of the distribution. Hence, the VaR at confidence level $\alpha$ is the negative of the (1- $\alpha)$-quantile, and typical values for $\alpha$ are $0.95,0.99$ or 0.995. The changes in the portfolio value are. $\sum_{i=1}^{n} \theta_{i} X_{i}$ where $\theta_{i}>0$ and $\sum_{i=0}^{n} \theta_{1}=1$.

In the sequel, we confine the analysis to the bivariate case, where the value of portfolio losses is given by $Y=\theta_{1} X_{1}+\theta_{2} X_{2}$, with $X_{i} \sim N\left(\mu_{i}, \sigma_{i}^{2}\right), \mathrm{i}=1,2$. The probability that $\mathrm{Y}$ is less than $\mathrm{q}$ is computed as $P(q)=\int_{-\infty}^{q} \int_{-\infty}^{+\infty} \phi\left(N\left(\frac{x_{1}-\mu_{1}}{\sigma_{1}}\right), N\left(\frac{y-\theta_{1} x_{1}-\theta_{2} \mu_{2}}{\theta_{2} \sigma_{2}}\right)\right) \exp \left[-\frac{\left(x_{1}-\mu_{1}\right)^{2}}{2 \sigma_{1}^{2}}\right]$ $\exp \left[-\frac{\left(y-\theta_{1} x_{1}-\theta_{2} \mu_{2}\right)^{2}}{2 \theta_{2}^{2} \sigma_{2}^{2}}\right] \frac{d x_{1} d y}{2 \pi \sigma_{1} \sigma_{2}}$.

Here, $\phi\left(u_{1}, u_{2}\right)$ denotes the copula density function. 
Changing to variables $\frac{x_{1}-\mu_{1}}{\sigma_{1}}=\hat{x}_{1}$ and denoting $\theta_{1} \mu_{1}+\theta_{2} \mu_{2}=: \mu$ and $\sum_{i=1}^{2} \theta_{i}^{2} \sigma_{i}^{2}=: \Xi^{2}$, the expression above is rewritten as $\int_{-\infty}^{q} \int_{-\infty}^{+\infty} \phi\left(N\left(\hat{x}_{1}\right), N\left(\frac{y-\theta_{1} \sigma_{1} \hat{x}_{1}-\mu}{\theta_{2} \sigma_{2}}\right)\right) \exp \left[-\frac{\hat{x}_{1}^{2}}{2}\right] \exp \left[-\frac{\left(y-\mu-\theta_{1} \sigma_{1} \hat{x}_{1}\right)^{2}}{2 \theta_{2}^{2} \sigma_{2}^{2}}\right]$ $\frac{d \hat{x}_{1} d y}{2 \pi \theta_{2} \sigma_{2}}$.

A further change of variables $\frac{y=\mu}{\Xi}=: v, \frac{\hat{x}_{1} \Xi}{\theta_{2} \sigma_{2}}-\frac{(y=\mu)}{\Xi} \frac{\theta_{1} \sigma_{1}}{\theta_{2} \sigma_{2}}=: z$ transforms the integral into

$$
p(q)=\int_{-\infty}^{\frac{q-\mu}{\Xi}} \int_{-\infty}^{+\infty} \phi\left(N\left(\frac{\nu \theta_{1} \sigma_{1}+z \theta_{2} \sigma_{2}}{\Xi}\right), N\left(\frac{\nu \theta_{2} \sigma_{2}+z \theta_{1} \sigma_{2}}{\Xi}\right)\right) \exp \left[-\frac{v^{2}+z^{2}}{2}\right] \frac{d z d v}{2} 2 \pi
$$

Note that an alternative expression for $\mathrm{P}(\mathrm{q})$ is.

$$
\iint_{\theta_{1} \sigma_{1} \hat{x}_{1}+\theta_{2} \sigma_{2} \hat{x}_{2} \leq q-\mu} \phi\left(\hat{x}_{1}, \hat{x}_{2}\right) \exp \left[-\frac{\hat{x}_{1}^{2}+\hat{x}_{2}^{2}}{2}\right] \frac{d \hat{x}_{1} d \hat{x}_{2}}{2 \pi}
$$

Assume the stochastic variables $X_{i}$ are deformed under ambiguity according to the Choquet framework of section " 5 . Conclusion", and let $c \in(0,0.5)$ denote the ambiguity parameter, as usual. Then, with obvious notation, $\mu^{(c)}=\mu^{(0.5)}+(2 c-1) \sum_{i=1}^{2} \theta_{i} \sigma_{i}$ and $\Xi^{(c)}$ $=2 \sqrt{c(1-c)} \Xi^{(0.5)}$.

Note that the last two expressions for $\mathrm{P}(\mathrm{q})$ show that the integral value is affected by the ambiguity parameter only throughout the integration set, while the integrand remains unchanged across different levels of $c$. This remark yields the following result.

\section{Proposition 2}

In the above-described setting, the relationship $V_{\alpha}(c)<V_{\alpha}(0.5)$ between the VaR at confidence level $\alpha$ under ambiguity level c, $0<\mathrm{c}<0.5$, and in absence of ambiguity is equivalent to.

$$
q_{1-\alpha}^{(0.5)}<\frac{1+2 \sqrt{c(1-c)}}{2 c-1} \sum_{1=1}^{2} \theta_{i} \sigma_{i}
$$

where $q_{1-\alpha}$ is the solution to $\mathrm{P}(q)=1-\alpha$ and $\mathrm{P}(q)$ is given by (4.1).

\section{Proof}

The VaR for the portfolio above at confidence level $\alpha$ is $-q_{1-\alpha}$, where $q_{1-\alpha}$ is the solution of the eq. $\mathrm{P}\left(q_{1-\alpha}\right)=\alpha$. Therefore.

$$
\left(q_{1-\alpha}^{(c)}-(2 c-1) \sum_{i=1}^{2} \theta_{1} \sigma_{1}\right) / \Xi^{(c)}=q_{1-\alpha}^{(0.5)} / \Xi^{(0.5)}
$$

which yields the relationship above in view of $\Xi^{(c)}=2 \sqrt{c(1-c)} \Xi^{(0.5)}$.

In the case of a Gaussian copula, this relationship boils down to the one we obtained in Remark 1. Indeed, the $\mathrm{VaR}$ has a well-known explicit expression. If we replace $\phi$ in (4.2) with the Gaussian density copula with correlation parameter $\rho$ and change to variables $\theta_{1} \sigma_{1} \hat{x}_{1}+\theta_{2} \sigma_{2} \hat{x}_{2}=\Xi_{\rho} y$ and $\hat{x}_{1} \Xi_{\rho}=z \sqrt{1-\rho^{2}} \theta_{2} \sigma_{2}+y\left(\rho \theta_{2} \sigma_{2}+\theta_{1} \sigma_{1}\right)$ with $\Xi_{\rho}$ $=\left(\sum_{i=1}^{n} \theta_{i}^{2} \sigma_{i}^{2}+2 \rho \sum_{i \neq j} \theta_{i} \theta_{j} \sigma_{i} \sigma_{j}\right)^{1 / 2}$, then (4.2) is written as $N\left(\frac{q-\mu}{\Xi_{\rho}}\right)$, i.e. $q_{1-\alpha}=\mu+\Xi_{\rho} N^{-1}(1-\alpha)$. 
However, in general, $q_{1-\alpha}$ cannot be represented through analytical expressions and is often computed by Montecarlo methods. Some studies have provided bounds for the numerical values of VaR obtained through copula methods (Embrechts et al. 2003).

In any case, Proposition 2 shows that the results we found in the Gaussian environment are preserved in a more general setting, at least at a qualitative level.

As an illustration, we perform simulations for three different copula functions and compute $\mathrm{VaR}$ at the $99 \%$ confidence level. We consider a portfolio of two assets with equal weight and with normal marginals, with a volatility of $10 \%$ for the holding period under consideration. The correlation is fixed at 0.7 , and the following copulas are

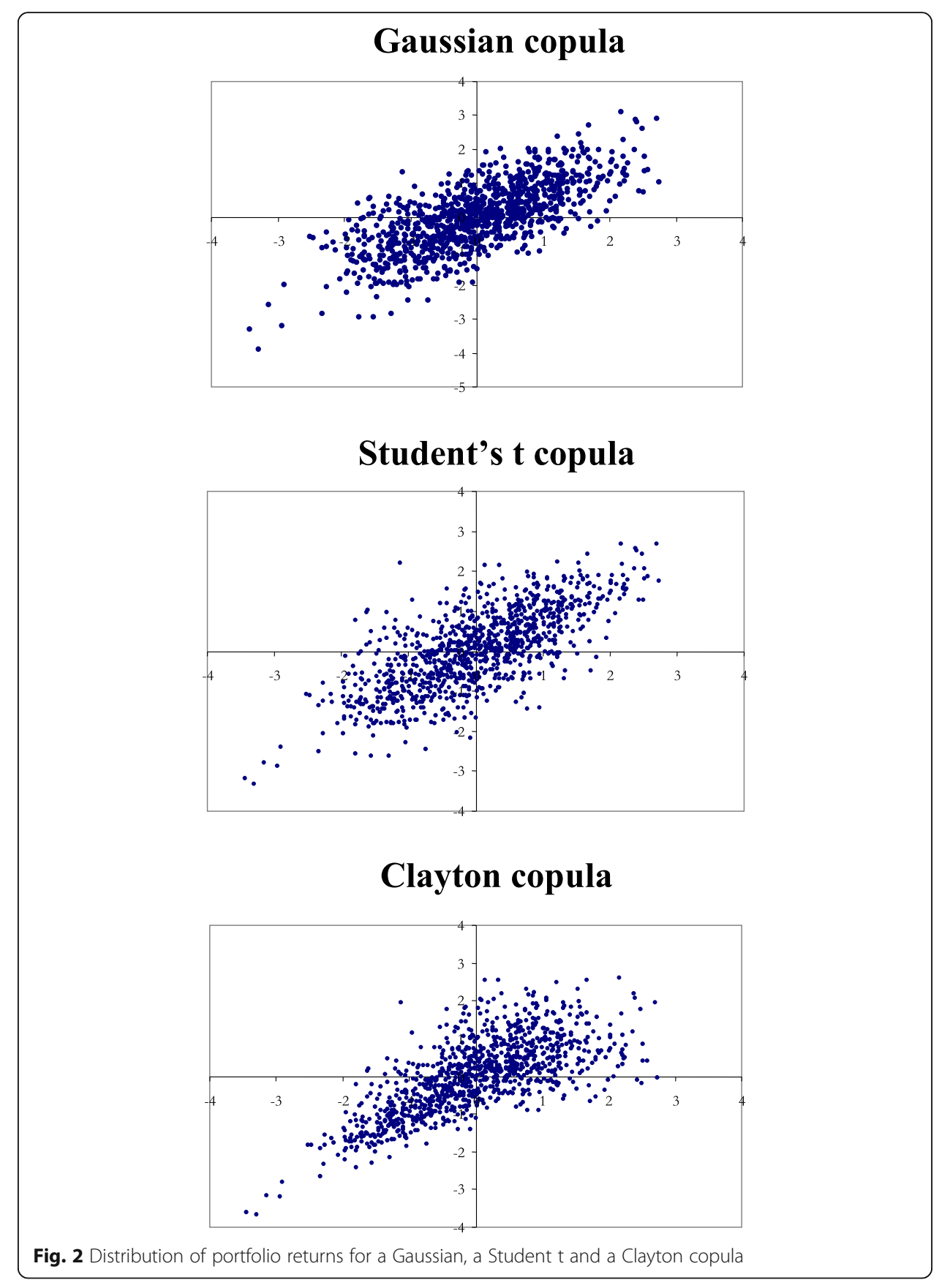


Table 6 Value-at-Risk at 99\% for a portfolio of two assets

\begin{tabular}{llllll}
\hline Copula & $\mathrm{c}=0.5$ & $\mathrm{c}=0.4$ & $\mathrm{c}=0.3$ & $\mathrm{c}=0.2$ & $\mathrm{c}=0.1$ \\
\hline Gaussian & $23.35 \%$ & $24.88 \%$ & $25.40 \%$ & $24.68 \%$ & $22.01 \%$ \\
Student & $23.45 \%$ & $24.98 \%$ & $25.50 \%$ & $24.76 \%$ & $22.07 \%$ \\
Clayton & $25.22 \%$ & $26.71 \%$ & $27.12 \%$ & $26.18 \%$ & $23.13 \%$ \\
\hline
\end{tabular}

adopted: a Gaussian one, a Student's $t$ with $v=5$ degrees of freedom and a Clayton copula with the parameter $a=2$. Figure 2 plots the distribution of the portfolio returns for the different forms of aggregation. Table 6 reports the computation of $\mathrm{VaR}$ at the $99 \%$ confidence level. Despite the slightly different values obtained from the three copulas, the phenomenon presented in Proposition 2 is evident across the alternative choices for the copula function.

\section{Conclusion}

This study investigated the effect of biases induced by ambiguity on the calculation of the most popular risk measure. Whereas any additional risk factor is expected to increase the estimated value of a risk measure, contrasting outcomes are possible when ambiguity comes into play. This confirms the different nature of ambiguity when compared to probabilistic risks. Broadly speaking, the results we obtained for individual assets were preserved at an aggregate level, and the imposed dependence structure did not seem to play a significant role. We chose to illustrate our results using the case of normal marginals, because a simple model for ambiguity-induced distortions is available, and the sensitivity of the risk measure stemming from biased marginal distributions can be obtained in a straightforward way. Alternative distributions can be used after a preliminary study of their behavior in an ambiguous environment.

As this study has shown, ambiguity aversion has a significant effect on risk measurement calculation. $\mathrm{T}$ is especially relevant in turbulent economic times. As mentioned in the Introduction, the level of ambiguity aversion can be calibrated to real-world data. Thus our results provide some practical guidelines for incorporating this singular form of uncertainty into relevant risk measures. The importance of understanding the effect of ambiguity on common risk measures cannot be overstated given their extensive use in internal risk management practices and prudential regulation.

\section{Endnotes}

${ }^{1}$ Equivalence holds for continuous distribution functions

${ }^{2}$ This assumption is adopted in the LHP model (Vasicek 1987) that has become a standard for credit risk assessment of large portfolios.

Acknowledgements

All scholars whose publications were useful for this work are listed in the reference list and are properly acknowledged in my paper. The professional writing service and the preparation of the numerical codes has been made by myself (the author).

Availability of data and materials

I confirm that the materials used for this work can be made available upon request. Issues related to data availability are not applicable.

Authors' contributions

The author read and approved the final manuscript. 
Ethics approval and consent to participate

I confirm my approval of the ethics of the journal and the publisher.

\section{Consent for publication}

I confirm my consent for publication.

\section{Competing interests}

The author declare that there are no competing interests.

\section{Publisher's Note}

Springer Nature remains neutral with regard to jurisdictional claims in published maps and institutional affiliations.

Received: 25 November 2017 Accepted: 4 June 2018

Published online: 13 June 2018

\section{References}

Agliardi E, Agliardi R, Spanjers W (2016) Corporate financing decisions under ambiguity: pecking order and liquidity policy implications. J Bus Res 69:6012-6020

Agliardi R (2017) Asymmetric Choquet random walks and ambiguity aversion or seeking. Theor Decis 83:591-602 Artzner P, Delbaen F, Eber JM, Heath D (1999) Coherent risk measures. Math Financ 9(3):203-228

Basel Committee on Banking Supervision (2013) Fundamental review of the trading book: a revised market risk framework. In: Second Consultative Paper, p 2013

Boyarchenko, N. (2010). 'Ambiguity shifts and the 2007-2008 financial crisis'. Mimeo

Driouchi T, Trigeorgis L, Gao Y (2015) Choquet-based European option pricing with stochastic (and fixed) strikes. Oper Res Spectrum 37:787-802

EIOPA-14-322, 2014 The underlying assumptions in the standard formula for the Solvency Capital Requirement calculation'

Embrechts P, Hoeing A, Juri A (2003) Using copulae to bound the value-at-risk for functions of dependent risks. Finance Stochast 7(2):145-167

Embrechts P, McNeil AJ, Straumann D (2002) Correlation and dependence in risk management: properties and pitfalls. In: Dempster M (ed) Risk Management: Value at Risk and Beyond. Cambridge University Press, Cambridge

Epstein L, Schneider M (2010) Ambiguity and asset markets. Ann Rev Financ Econ 2:315-346

Ghamani S (2015) Static models of central counterparty risk. J Financ Eng 2(n 1550011):36

Guidolin M, Liu H (2016) Ambiguity aversion and under-diversification. J Financ Quant Anal 51(4):1297-1323

Gupton GM, Finger CC, Bhatia M (1997) CreditMetricsTM - technical document. J. P. Morgan \& Co., New York

Hens T, Rieger MO (2014) Can utility optimization explain the demand for structured investment products? Quant Fin 14(4):673-681

Kast R, Lapied A (2010a) Valuing future cash flows with non separable discount factors and non additive subject measures: conditional Choquet capacities on time and uncertainty. Theor Decis 69(1):27-53

Kast, R. \& A. Lapied (2010b). Dynamically consistent Choquet random walk and real investments, Document de Travail n. 2010-33, GREQAM, HAL id: halhs-00533826

Nelsen R (1999) An introduction to copulas. In: Lecture Notes in Statistics. Springer, N.Y., p 139

Rieger MO, Wang M, Hens T (2015) Risk preferences around the world. Manag Sci 61(3):637-648

Schmeidler D (1989) Subjective probability and expected utility without additivity. Econometrica 57(3):571-587

So, R. H. Y. (2017). The role of ambiguity in financial markets: applications to returns, volatility, and economic predictions. King's College London, PhD thesis

Trautman S, Van de Kuilen G (2013) Ambiguity attitudes. In: Keren G, Wu G (eds) Blackwell Handbook of Judgment and Decision Making. Blackwell, Oxford

Turner Review (2009). A regulatory response to the global banking crisis, published by the British Financial Services Authority, www.fsa.gov.uk/pubs/other/turner_review.pdf, 2009 